\title{
Update bietet Usern viele neue Funktionen
}

Ende des vergangenen Jahres wurde die Röntgensoftware SIDEXIS 4 für ihre herausragende Nutzerfreundlichkeit mit dem international renommierten Red Dot Award Best of the Best in der Kategorie "Communication Design" ausgezeichnet. Das neue Software-Update 4.1 .3 von Dentsply Sirona Imaging kombiniert jetzt zusätzliche Funktionalitäten für Anwender mit technischen Änderungen: Verbes-

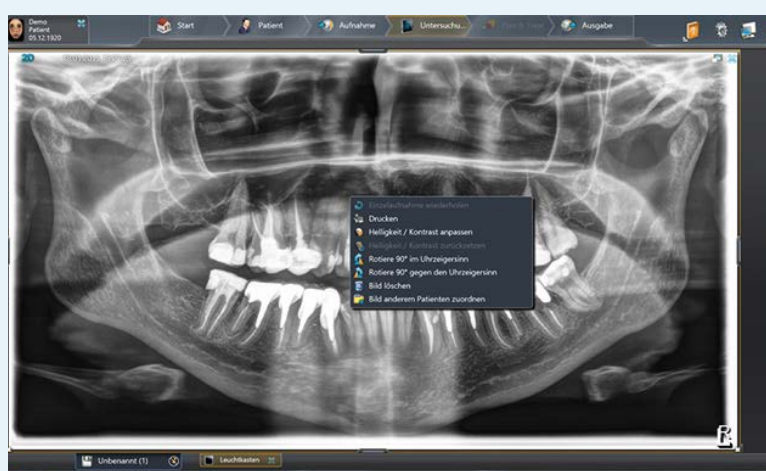

serte Performance, Integration der SICAT Suite oder Fremdkamera-Anbindung: Das Software-Update ermöglicht dem Anwender viele neue Funktionalitäten. Mit einem neuen SQL Server, der Kompatibilität mit Windows 10 und weiteren Betriebssystemen wurden auch die technischen Funktionen erweitert. Vor allem in Kombination mit dem ORTHOPHOS SL bildet die SIDEXIS 4 Software eine höchst funktionelle und effiziente Einheit. Das Update bietet zudem die Vorteile bei der Vernetzung mit Praxisverwaltungsprogrammen sowie implantologischen Planungs- oder kieferorthopädischen Analyseprogrammen.

Nach einer Pressemitteilung der

Dentspla Sirona Imaging, Bensheim 